\title{
Landing Impact Analysis of a Bioinspired Intermittent Hopping Robot with Consideration of Friction
}

\author{
Long Bai, ${ }^{1}$ Wenjie Ge, ${ }^{2}$ Xiaohong Chen, ${ }^{1}$ Qian Tang, ${ }^{1}$ and Rong Xiang ${ }^{3}$ \\ ${ }^{1}$ State Key Laboratory of Mechanical Transmissions, Chongqing University, Chongqing 400044, China \\ ${ }^{2}$ School of Mechanical Engineering, Northwestern Polytechnical University, Xian 710072, China \\ ${ }^{3}$ Chongqing Wushan Power Supply Co., Ltd., State Grid Corporation of China, Chongqing 404700, China \\ Correspondence should be addressed to Long Bai; bailong@cqu.edu.cn
}

Received 6 August 2014; Revised 10 November 2014; Accepted 14 November 2014

Academic Editor: Shaoping Bai

Copyright (C) 2015 Long Bai et al. This is an open access article distributed under the Creative Commons Attribution License, which permits unrestricted use, distribution, and reproduction in any medium, provided the original work is properly cited.

\begin{abstract}
Multibody dynamics for the flying and landing oblique impact processes of a bioinspired intermittent hopping robot is derived in this paper by using the impulse-momentum principle. The dynamics model that involves the multibody configuration, mass distribution of the robot, and friction is solved by the linear complementarity conditions in terms of different impact types. The computational and experimental data is compared. And the influence factors of landing impact are analysed as well. Based on the influence rules for landing impact, a technical design of solution is proposed for adjusting the robot's attitude during the jumping and for absorbing the impact energy during the landing. Lessons learned from the theoretical and experimental results have general applicability to the motion prediction, performance analysis, and landing stability study for intermittent hopping robots or other legged robots.
\end{abstract}

\section{Introduction}

Legged locomotion, especially "hopping" or "jumping," has better mobility in unknown, rugged terrains and low/microgravity environments $[1,2]$. It can overcome the drawbacks of wheeled rovers, such as limitations on flexibility of locomotion, ability to traverse, and miniaturization [2-7]. Hence, hopping robotics has become one of the focuses in the fields such as planetary surface exploration, rescue operation, and reconnaissance [8-15].

In consideration of practicality, most of hopping robots are intermittent hopping paradigms, since a pause for reorientation and recharge of the hopping mechanism of paradigms must be inserted between hops/jumps. To date, most previous works on intermittent hopping robots concentrate on mechanical design and implementation. By mimicking the jumping characteristics of animals, such as frog and locust, they mainly focus on how to jump up and how to jump higher or longer, such as "three generation hoppers" [11, 12], "MINERVA" [13, 14], and "MICROBOTS" [15]. However, they are still simple catapult mechanisms, with a single and unstable hopping stance. As a consequence of this, they can realize hopping movement, but their bodies always hit the ground sharply and land in an unpredictable toppled stance. Although it can upright itself with a standing posture by self-recovery mechanism after landing, the unstable flying posture and explosive landing impact will go against the solution of landing stability and even break the devices of the robots. Given this, some other intermittent hopping paradigms turn to "multimobile robotic systems", which employs wheeled-jumping or jump-gliding, such as "Portable Guard Robot" [16, 17], "Leg-in-Rotor" [18, 19], "EPFL Robot" [20], and "Flying Fish Glider" [21]. These robotic systems could protect the hopping mechanisms from hitting the ground directly. However, they also have negative aspects like damage to wheels, increased tractive resistance, and so forth.

As such, landing impact of intermittent hopping robots is a pressing issue to be researched. Lessons learned from the landing impact analysis have general applicability to intermittent hopping robot and will provide technical reference to legged robotic systems as well. But until now, few specific studies on this topic are presented in reports and mostly are the normal impact dynamic analysis of the intermittent hopping robots by simplifying the robots 


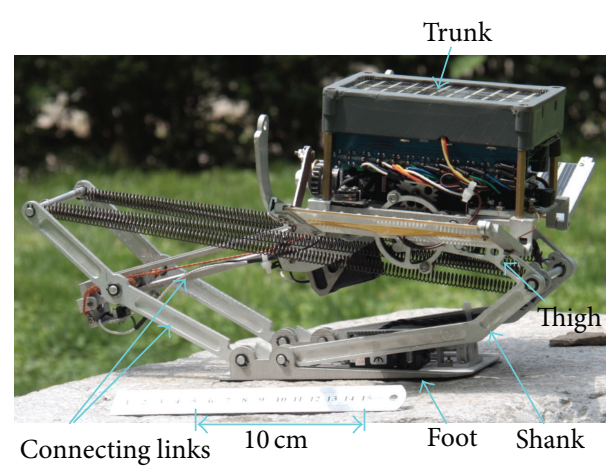

FIGURE 1: Prototype of the proposed robot [17], bioinspired by kangaroo, which is bipedal with a synchronous hopping motion along the sagittal plane of its body.

as nonlinear spring-mass models $[22,23]$. The simplified dynamics models and their analysis have not yet involved multibody dynamics and friction, which are unable to reveal the practical landing characteristics of hopping mechanisms, such that they are inapplicable to the motion prediction and performance analysis of intermittent hopping robots.

Our prior work designed a small, bioinspired, and minimally actuated intermittent hopping robot (as shown in Figure 1) [24] which is suitable for exploring the unstructured terrains of celestial bodies. The robot uses a novel hopping mechanism, combined geared six-bar linkages and springs, which has a possible rich trajectory and metamorphic characteristics. The mechanical design and the constrained multibody dynamics for the take-off process principle of the proposed robot are presented in [24]. The theoretical analysis and prototype experimental results show that the proposed robot is able to recharge, lock/release, and jump by using only one micropower motor as the actuator and can achieve a biomimetic multibody hopping stance and a nonlinearly increasing driving force. Based on the prior work, this paper turns to further study the landing characteristics and the influencing factors of the proposed robot and aims to provide general applicability to motion prediction and landing performance analysis of intermittent hopping robots or legged robots. The multibody dynamics models for flying phase and landing oblique impact are firstly derived by taking the multibody configuration, mass distribution of the robot, friction, and so forth into consideration. Next, the established dynamics models are solved in terms of impact types. Finally, the influence factors of landing characteristics are analysed, and a technical design solution is proposed as well.

\section{Hopping Process of the Proposed Robot}

The proposed robot can change its mechanical structure and DOF (degree of freedom) in steps to recharge the hopping mechanism, upright itself after landing, and point itself in the desired direction before take-off by utilizing its metamorphic characteristics. When the robot is ready to jump, the recharged springs are released, whereupon take-off commences. The follow-up is the flying and landing process.

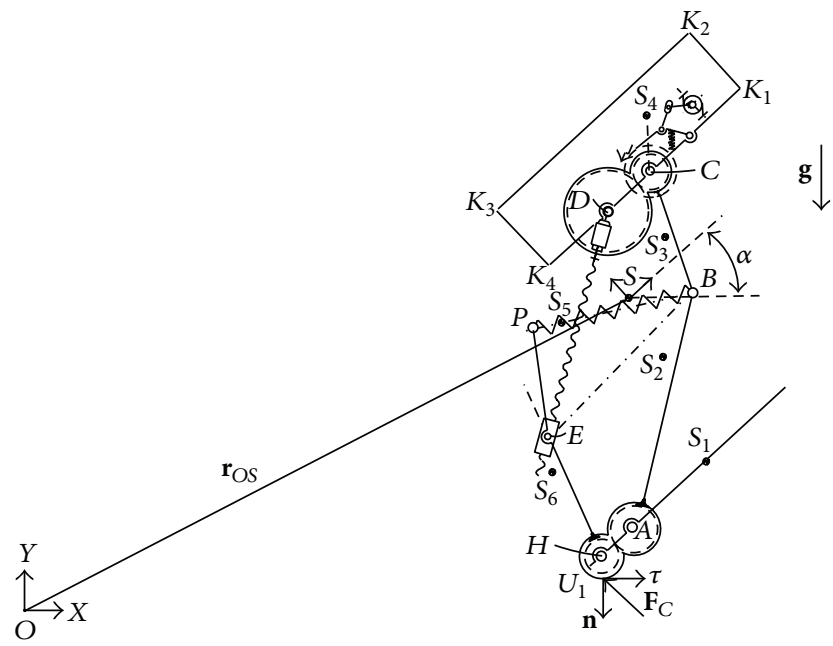

FIGURE 2: Dynamics analysis model of the proposed robot, where $O X Y$ ( $O$ on the ground) is the fixed-coordinate system, $S$ is the centre of mass (COM) of the robot, $S_{1}, \ldots, S_{6}$ are the COM of parts of the robot, respectively, $A, \ldots, E, H$ are the joints of the robot, respectively, $K_{1}, \ldots, K_{4}$ are the boundary points of trunk, and $g$ is the gravitational acceleration.

When the robot begins to take off, its topological structure is changed into $6 \mathrm{R}-2 \mathrm{G}$ (R and G represent revolute joint and gear pair, resp.) with 1-DOF until landing, where the robot is a passive system without active control. Subjected to the force of springs, the parts of the robot can be considered to be without relative motion to each other. Accordingly, after take-off, the robot can be considered as an irregular rigidbody, whose COM (centre of mass) moves along a parabolic trajectory and parts move relative to COM with inertial motion. When the robot is landing, the rear end of its sole is the first to drop to ground, and then the robot hits the ground and bounces off. The follow-up is alternating impact and bounces on and on until the robot stops on the ground.

Hence, the dynamics analysis model of the flying and landing process can be presented as shown in Figure 2. By referring to the movement of the kangaroo, the proposed robot, based on a geared six-bar parallel mechanism, is bipedal with a synchronous (similar to a single leg, shown in Figure 1) hopping motion along the sagittal plane of its body. As such, its flying and landing motion should lie in the same sagittal plane as well, if there are no crosswind, rugged terrain, and so forth. Though, of course, these idealized conditions are not easy to completely reach, plane motion is still the typical characteristic of the flying and landing process since the robot is a projectile without active control during flying and landing. Accordingly, here the lateral motion of the robot is neglected; that is, the motion in $Z$-direction (perpendicular to the surface of the paper) is neglected.

\section{Dynamics Modelling of the Flying Process}

As shown in Figure 2, the flying process of the robot is plane motion of a rigid-body. During flying, the position and the attitude of the robot at any given time are determined by $\mathbf{r}_{O S}$ (the position vector of the robot's COM $S$ in the fixedcoordinate system $O X Y$ ) and attitude angle $\alpha$ (the rotation 
angle of the moving coordinate system on $S$ relative to $O X Y)$. Hence, $\mathbf{q}=\left[\mathbf{r}_{\mathrm{OS}}, \alpha\right]^{T}=[x, y, \alpha]^{T}$ is selected as the generalized coordinates, where $x$ and $y$ are displacements of $S$ in $X$-direction and $Y$-direction, respectively. Accordingly, the position vector and rotation velocity of $S$, namely, $\mathbf{r}_{O S}$ and $\boldsymbol{\omega}$, can be expressed by [25]

$$
\mathbf{r}_{\mathrm{OS}}=\left[\begin{array}{lll}
x & y & 0
\end{array}\right]^{T}, \quad \boldsymbol{\omega}=\left[\begin{array}{lll}
0 & 0 & \dot{\alpha}
\end{array}\right]^{T} .
$$

By taking partial derivative of $\mathbf{r}_{O S}$ and $\boldsymbol{\omega}$ with respect to $\mathbf{q}$ and $\dot{\mathbf{q}}$, respectively, the translational matrix $\mathbf{J}_{\mathrm{TS}}$ and the rotational Jacobian matrix $\mathbf{J}_{\mathrm{RS}}$ can be obtained as

$$
\mathbf{J}_{\mathrm{TS}}=\frac{\partial \mathbf{r}_{\mathrm{OS}}}{\partial \mathbf{q}}=\left[\begin{array}{lll}
1 & 0 & 0 \\
0 & 1 & 0 \\
0 & 0 & 0
\end{array}\right], \quad \mathbf{J}_{\mathrm{RS}}=\frac{\partial \boldsymbol{\omega}}{\partial \dot{\mathbf{q}}}=\left[\begin{array}{lll}
0 & 0 & 0 \\
0 & 0 & 0 \\
0 & 0 & 1
\end{array}\right]
$$

Since the robot is only subjected to gravity during flying, it can be defined as a time-invariant holonomic rigid-body dynamics system, such that its dynamics model can be expressed by

$$
\mathbf{M}(t, \mathbf{q}) \ddot{\mathbf{q}}+\mathbf{K}(t, \mathbf{q}, \dot{\mathbf{q}})=\mathbf{J}_{\mathrm{TS}}^{T} \mathbf{f}^{e}+\mathbf{J}_{\mathrm{RS}}^{T} \mathbf{l}^{e},
$$

where $\mathbf{M}(t, \mathbf{q}) \in R^{3 \times 3}$ is the positive definite mass matrix, $\mathbf{K}(t, \mathbf{q}, \dot{\mathbf{q}}) \in R^{3}$ is the generalized gyroscopic force, centrifugal force, and Coriolis force, and $\mathbf{f}^{e}$ and $\mathbf{l}^{e} \in R^{3}$ are the generalized active force and active torque.

Let $\mathbf{I}_{S}$ be the inertia tensor of the robot on $S$ and let $m$ be the mass of the robot; that is,

$$
m=\sum_{i=1}^{6} m_{i} \quad(\text { see }[24]) .
$$

Since parts of the robot are without relative motion to each other, $\mathbf{M}(t, \mathbf{q})$ and $\mathbf{K}(t, \mathbf{q}, \dot{\mathbf{q}})$ can be expressed by

$$
\begin{gathered}
\mathbf{M}(t, q)=\mathbf{J}_{\mathrm{TS}}^{T} m \mathbf{J}_{\mathrm{TS}}+\mathbf{J}_{\mathrm{RS}}^{T} \mathbf{I}_{S} \mathbf{J}_{\mathrm{RS}}, \\
\mathbf{K}(t, q, \dot{q})=\mathbf{J}_{\mathrm{RS}}^{T} \widetilde{\boldsymbol{\omega}}_{S} \mathbf{I}_{S} \boldsymbol{\omega}_{S},
\end{gathered}
$$

where

$$
\widetilde{\boldsymbol{\omega}}_{S}=\left[\begin{array}{ccc}
0 & -\dot{\alpha} & 0 \\
\dot{\alpha} & 0 & 0 \\
0 & 0 & 0
\end{array}\right]
$$

Since gravity is the only external force acting on $S, \mathbf{f}^{e}$ and $\mathbf{l}^{e}$ can be expressed by

$$
\mathbf{f}^{e}=[0,-m g, 0]^{T}, \quad \mathbf{l}^{e}=[0,0,0]^{T} .
$$

\section{Dynamics Modelling of the Landing Process}

4.1. Dynamics Equations of the Landing Process. When the robot begins to land, impact commences between the robot and the ground. Define $U_{1}$ as the impact point on the robot, $U_{2}$ as the corresponding impact point on the ground, and $\mathbf{F}_{C}$ as the impact force subjected by $U_{1}$. Since the robot will typically land nonperpendicularly to the ground, $\mathbf{F}_{C}$ can be divided into 2 component forces as follows:

$$
\mathbf{F}_{C}=\lambda_{\tau}(t) \boldsymbol{\tau}+\lambda_{N}(t) \mathbf{n},
$$

where $\lambda_{\tau}(t)$ and $\lambda_{N}(t)$ are the scalar values of tangential and normal impact forces, respectively, $\mathbf{n}$ is the unit normal vector at the point $U_{2}$ (from the ground points to the robot), $\mathbf{n}=[0,1,0]^{T}, \boldsymbol{\tau}$ is the unit vector in direction of tangential velocity of the point $U_{1}$ at initial moment of landing, and

$$
\boldsymbol{\tau}=\frac{\dot{\mathbf{r}}^{C}-\mathbf{n}^{T} \dot{\mathbf{r}}^{C} \mathbf{n}}{\left|\dot{\mathbf{r}}^{C}-\mathbf{n}^{T} \dot{\mathbf{r}}^{C} \mathbf{n}\right|}
$$

where $\dot{\mathbf{r}}^{\mathrm{C}}$ is the relative velocity between $U_{1}$ and $U_{2}$ at initial moment of landing.

Since the landing impact process is extremely short, the relative positions of parts of the robot can be considered as remaining unchanged; that is, the configuration of the robot remains time-invariant. Here, assume that there is only one touching point when the robot impacts the ground. Since the robot is only subjected to the external active force $\mathbf{f}^{e}$ and impact force $\mathbf{F}_{C}$, the landing impact dynamics equation can be expressed by

$$
\mathbf{M} \cdot \ddot{q}=\mathbf{J}_{\mathrm{TS}}^{T} \mathbf{f}^{e}+\mathbf{J}_{\mathrm{TU}}^{T} \cdot\left[\lambda_{\tau}(t) \boldsymbol{\tau}+\lambda_{N}(t) \mathbf{n}\right],
$$

where $\mathbf{M} \in R^{3 \times 3}$ is the positive definite mass matrix and $\mathbf{J}_{\mathrm{TU}}$ is the translational Jacobian matrix of $U_{1}$.

Let $t_{A}$ and $t_{E}$, respectively, be the starting time and ending time of landing impact process. Since the impact force is instantaneous and explosive, the other forces can be neglected by comparing with the impact force. As the impact period approximates zero, based on the law of conservation of momentum, the integration of (10) with respect to $t$ in the interval $\left[t_{A}, t_{E}\right]$ can be obtained as

$$
\mathbf{M} \cdot\left(\dot{\mathbf{q}}_{E}-\dot{\mathbf{q}}_{A}\right)-\mathbf{w}_{\tau} \cdot \boldsymbol{\Lambda}_{\tau}-\mathbf{w}_{N} \cdot \boldsymbol{\Lambda}_{N}=0,
$$

where $\dot{\mathbf{q}}_{E}$ and $\dot{\mathbf{q}}_{A}$ are the values of generalized coordinates at times $t_{A}$ and $t_{E}$, respectively, $\boldsymbol{\Lambda}_{\tau}$ and $\boldsymbol{\Lambda}_{N}$ are the impulses generated by the tangential and normal impact forces during landing impact process, and

$$
\begin{array}{cc}
\mathbf{w}_{N}=\mathbf{J}_{\mathrm{TU}}^{T} \cdot \mathbf{n}, \quad \mathbf{w}_{\tau}=\mathbf{J}_{\mathrm{TU}}^{T} \cdot \boldsymbol{\tau}, \\
\boldsymbol{\Lambda}_{\tau}=\lim _{t_{E} \rightarrow t_{A}} \int_{t_{A}}^{t_{B}} \lambda_{\tau} \mathrm{d} t, \quad \boldsymbol{\Lambda}_{N}=\lim _{t_{E} \rightarrow t_{A}} \int_{t_{A}}^{t_{B}} \lambda_{N} \mathrm{~d} t .
\end{array}
$$

According to the geometrical relationship of contact between rigid-bodies [25], $\dot{\mathbf{r}}$, the relative velocity between $U_{1}$ and $U_{2}$, can be obtained by

$$
\dot{\mathbf{r}}=\mathbf{J}_{\mathrm{TU}} \dot{\mathbf{q}}
$$

4.2. Analysis of the Landing Impact Process. In a single landing impact process, depending upon the tangential velocity of the robot at the moment that impact occurs, as well as the conditions of ground (coefficient of friction, coefficient 


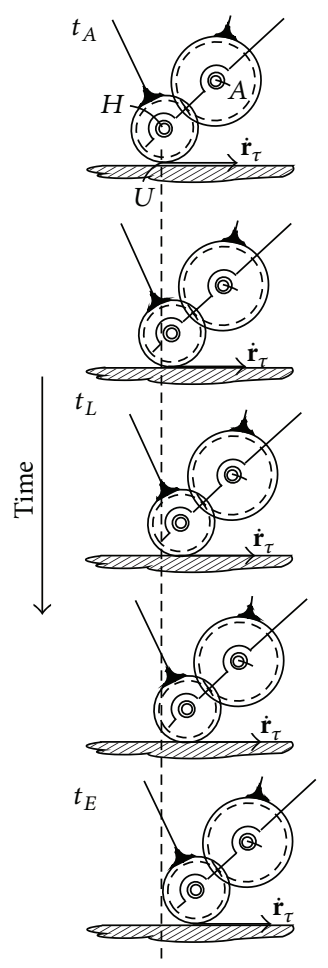

(a) USI

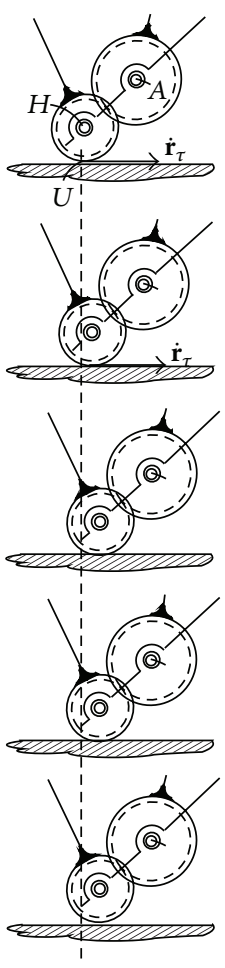

(b) VI

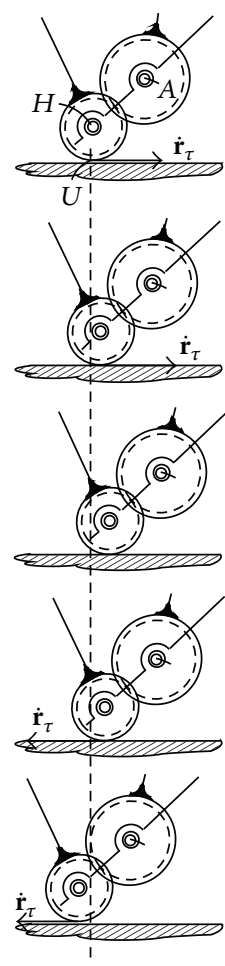

(c) RSI

FIGURE 3: Three types of the robot during landing impact, where the top-to-down subfigures of each column, respectively, show the statuses at different time in each type of impact process.

of restitution, etc.), $\dot{\mathbf{r}}_{\tau}$ (the tangential relative motion between $U_{1}$ and $U_{2}$ ) has three possible cases as shown in Figure 3.

According to the three possible cases, the impact process can be classified into three types-unidirectional sliding impact (USI), viscous impact (VI), and reverse sliding impact (RSI) - which are characterized by the following $[25,26]$.

(1) USI: $\dot{\mathbf{r}}_{\tau}$ remains unchanged throughout the impact process.

(2) VI: in impact process, $\dot{\mathbf{r}}_{\tau}$ decreases gradually to zero at a certain moment $t_{L}$ and then remains zero.

(3) RSI: in impact process, $\dot{\mathbf{r}}_{\tau}$ decreases gradually to zero at a certain moment $t_{L}$ and then reverse sliding occurs.

Since the tangential impact force $\lambda_{\tau}(t) \tau$ is the Coulomb friction force, based on Coulomb's law of friction, the relationships between $\lambda_{\tau}(t)$ and $\lambda_{N}(t)$ can be obtained in terms of the three impact types as follows.

(1) USI:

$$
\lambda_{\tau}(t)=-\mu_{d} \lambda_{N}(t) \quad t \in\left(t_{A}, t_{E}\right) .
$$

(2) VI:

$$
\lambda_{\tau}(t)= \begin{cases}-\mu_{d} \lambda_{N}(t) & t \in\left(t_{A}, t_{L}\right) \\ -\mu_{s} \lambda_{N}(t) & t \in\left(t_{L}, t_{E}\right) .\end{cases}
$$

(3) RSI:

$$
\lambda_{\tau}(t)= \begin{cases}-\mu_{d} \lambda_{N}(t) & t \in\left(t_{A}, t_{L}\right) \\ \mu_{d} \lambda_{N}(t) & t \in\left(t_{L}, t_{E}\right),\end{cases}
$$

where $t_{L}$ is the time when $\dot{\mathbf{r}}_{\tau}$ decreases to $0, \mu_{d}$ is the coefficient of sliding friction between the robot and the ground, and $\mu_{s}$ can be considered as the actual coefficient of static friction. Being different from the coefficient of static friction in usual meaning that is only relative with the maximum static friction force, the actual coefficient of static friction is the ratio of the real-time static friction force to the corresponding normal contact force. Equations (14) (16) can be expressed by a unified expression as follows:

$$
\lambda_{\tau}(t)=-\mu \lambda_{N}(t)
$$

where $\mu \in\left\{\mu_{d},-\mu_{d}, \mu_{s}\right\}$ is determined by the type of impact process and time $t$.

Let $\mu$ remain unchanged from $t_{1}$ to $t_{2}$. By integrating (17) with respect to $t$ in the interval $\left[t_{1}, t_{2}\right]$, the relationship between $\boldsymbol{\Lambda}_{\tau}$ and $\boldsymbol{\Lambda}_{N}$ can be obtained as

$$
\boldsymbol{\Lambda}_{\tau}=-\mu \boldsymbol{\Lambda}_{N}=-\mu \int_{t_{1}}^{t_{2}} \lambda_{N}(t) \mathrm{d} t .
$$


Let

$$
\begin{aligned}
& \mathbf{E}_{\tau}=\boldsymbol{\tau}^{T} \mathbf{J}_{\mathrm{TU}} \mathbf{M}^{-1} \mathbf{J}_{\mathrm{TU}}^{T} \cdot\left(\mathbf{n}-\mu_{d} \boldsymbol{\tau}\right), \\
& \mathbf{E}_{N}=\mathbf{n}^{T} \mathbf{J}_{\mathrm{TU}} \mathbf{M}^{-1} \mathbf{J}_{\mathrm{TU}}^{T} \cdot\left(\mathbf{n}-\mu_{d} \boldsymbol{\tau}\right) \\
& \mathbf{E}_{\tau}^{\prime}=\boldsymbol{\tau}^{T} \mathbf{J}_{\mathrm{TU}} \mathbf{M}^{-1} \mathbf{J}_{\mathrm{TU}}^{T} \cdot\left(\mathbf{n}+\mu_{d} \boldsymbol{\tau}\right), \\
& \mathbf{E}_{N}^{\prime}=\mathbf{n}^{T} \mathbf{J}_{\mathrm{TU}} \mathbf{M}^{-1} \mathbf{J}_{\mathrm{TU}}^{T} \cdot\left(\mathbf{n}+\mu_{d} \boldsymbol{\tau}\right) .
\end{aligned}
$$

In landing impact process, $\mu=\mu_{d}$ until $\dot{\mathbf{r}}_{\tau}$ decreases to 0 and $\boldsymbol{\Lambda}_{N} \geq 0$ once the impact/contact exists. So, if $\mathbf{E}_{\tau} \geq 0$, $\dot{\mathbf{r}}_{\tau}$ will remain $\dot{r}_{\tau}(t) \geq \dot{r}_{\tau}\left(t_{A}\right)>0$; that is, the direction of $\dot{\mathbf{r}}_{\tau}$ keeps unchanged. In this case, the status of impact is USI.

Conversely, if $\mathbf{E}_{\tau}<0, \dot{r}_{\tau}(t)$ decreases from $\dot{r}_{\tau}\left(t_{A}\right)$, and possibly to 0 . However, whether $\dot{r}_{\tau}(t)$ can decrease to 0 , it is determined by such factors, as the coefficient of friction and the velocity of the robot before landing impact occurs, and so forth. According to the status of the contact between the robot and the ground, the landing impact process can be divided into two phases, that is, the compression phase and the expansion phase. The compression phase proceeds from $t_{A}$ to the time $\dot{r}_{N}(t)$ decreases to 0 ; then, the expansion phase proceeds, until the impact process terminates. Here, let the compression phase be within the interval $\left[t_{A}, t_{C}\right]$ and let the expansion phase be within the interval $\left[t_{C}, t_{E}\right]$. By replacing the integral interval of (11) with $\left[t_{A}, t_{L}\right]$, the normal impulse $\boldsymbol{\Lambda}_{N, A L}$ along $\dot{r}_{\tau}(t)$ decreasing to 0 can be obtained as $\boldsymbol{\Lambda}_{N, A L}=$ $-1 / \mathbf{E}_{\tau} \dot{r}_{\tau}\left(t_{A}\right)$ and the normal impulse of the compression phase $\boldsymbol{\Lambda}_{N, A C}$ (along $\dot{r}_{N}(t)$ decreasing to 0 ) can be obtained as $\Lambda_{N, A C}=-1 / \mathbf{E}_{N} \dot{r}_{N}\left(t_{A}\right)$. Let $\varepsilon_{P}$ be the coefficient of restitution, which is the ratio of the normal impulse of the compression phase $\boldsymbol{\Lambda}_{N, A C}$ to the normal impulse of the expansion phase $\boldsymbol{\Lambda}_{N, C E}$. If $\boldsymbol{\Lambda}_{N, A L} \geq\left(1+\varepsilon_{P}\right) \boldsymbol{\Lambda}_{N, A C}$ [25], it indicates that $\dot{r}_{\tau}(t)$ is impossible to decrease to 0 . In this case, the impact process is USI. Accordingly, if $\boldsymbol{\Lambda}_{N, A L}<\left(1+\varepsilon_{P}\right) \boldsymbol{\Lambda}_{N, A C}$, it indicates that $\dot{r}_{\tau}(t)$ will decrease to 0 . When $\dot{r}_{\tau}(t)$ decreases to 0 , whether $\dot{r}_{\tau}(t)$ will reverse or remain $\dot{r}_{\tau}(t)=0$, it is determined by $\mathbf{E}_{\tau}^{\prime}$. By replacing the integral interval of (11) with $\left[t_{A}, t_{L}\right]$ and $\left[t_{L}, t\right]\left(t \in\left[t_{L}, t_{E}\right]\right)$, respectively, $\dot{r}_{\tau}(t)$ can be expressed by

$$
\dot{\mathbf{r}}_{\tau}(t)=\mathbf{E}_{\tau} \boldsymbol{\Lambda}_{N, A L}+\mathbf{E}_{\tau}^{\prime} \boldsymbol{\Lambda}_{N, L E}+\dot{\mathbf{r}}_{\tau}\left(t_{A}\right),
$$

where $\mathbf{E}_{\tau} \boldsymbol{\Lambda}_{N, A L}+\dot{\mathbf{r}}_{\tau}\left(t_{A}\right)=0$ and $\boldsymbol{\Lambda}_{N, L E} \geq 0$. Hence, if $\mathbf{E}_{\tau}^{\prime}<0$, $\dot{\mathbf{r}}_{\tau}(t)$ should be $\dot{\mathbf{r}}_{\tau}(t)<0$; that is, $\dot{r}_{\tau}(t)$ changes from 0 to negative value, whereupon the corresponding impact process is RSI. Conversely, if $\mathbf{E}_{\tau}^{\prime} \geq 0, \dot{\mathbf{r}}_{\tau}(t)$ should be $\dot{\mathbf{r}}_{\tau}(t) \geq 0$; that is, $\dot{r}_{\tau}(t)$ changes from 0 to positive value. However, since $E_{\tau}<$ 0 will prevent $\dot{r}_{\tau}(t)$ from increasing to positive value, $\dot{r}_{\tau}(t)$ remains 0 , whereupon the corresponding impact process is VI.

As the classified criterions of the types of impact process discussed above, the corresponding classified table can be summarized as in Table 1 .

4.3. Dynamics Models for Types of Landing Impact Process. As discussed above, in the landing impact process of the proposed robot, there are three possible cases depending upon the conditions of contact. Consequently, there should
TABLE 1: Classified table of the types of impact process.

\begin{tabular}{lccc}
\hline \multirow{2}{*}{ Criterions } & \multirow{2}{*}{$\mathrm{\tau} \geq 0$} & \multicolumn{2}{c}{$E \tau<0$} \\
& & $E^{\prime} \tau<0$ & $E^{\prime} \tau \geq 0$ \\
\hline $\boldsymbol{\Lambda}_{N, A L} \geq\left(1+\varepsilon_{P}\right) \Lambda_{N, A C}$ & USI & USI & USI \\
$\Lambda_{N, A L}<\left(1+\varepsilon_{P}\right) \Lambda_{N, A C}$ & USI & RSI & VI \\
\hline
\end{tabular}

be three corresponding dynamics models for the landing impact process.

Based on dynamics equations (11) and (13), as well as Coulomb's law of friction, $\mathbf{r}$ at any time in impact process can be obtained by integrating (10) with respect to $t$ in the interval $\left[t_{1}, t_{2}\right]$ as follows:

$$
\dot{\mathbf{r}}\left(t_{2}\right)=\mathbf{J}_{\mathrm{TU}} \mathbf{M}^{-1} \mathbf{J}_{\mathrm{TU}}^{T}(\mathbf{n}-\mu \tau) \boldsymbol{\Lambda}_{N}+\dot{\mathbf{r}}\left(t_{1}\right) .
$$

Accordingly, the tangential and normal components of $\dot{\mathbf{r}}, \dot{\mathbf{r}}_{\tau}$ and $\dot{\mathbf{r}}_{N}$, can be expressed by

$$
\begin{aligned}
\dot{\mathbf{r}}_{\tau}\left(t_{2}\right) & =\boldsymbol{\tau}^{T} \mathbf{J}_{\mathrm{TU}} \mathbf{M}^{-1} \mathbf{J}_{\mathrm{TU}}^{T} \cdot(\mathbf{n}-\mu \boldsymbol{\tau}) \boldsymbol{\Lambda}_{N}+\dot{\mathbf{r}}_{\tau}\left(t_{1}\right), \\
\dot{\mathbf{r}}_{N}\left(t_{2}\right) & =\mathbf{n}^{T} \mathbf{J}_{\mathrm{TU}} \mathbf{M}^{-1} \mathbf{J}_{\mathrm{TU}}^{T} \cdot(\mathbf{n}-\mu \boldsymbol{\tau}) \boldsymbol{\Lambda}_{N}+\dot{\mathbf{r}}_{N}\left(t_{1}\right) .
\end{aligned}
$$

In impact process, the surface of contact is only subjected to positive pressure and therefore $\lambda_{N}$ should be nonnegative, namely, $\lambda_{N} \geq 0$, as well as $\Lambda_{N} \geq 0$. Likewise, since there is no penetration between the robot and the ground in impact process, $\dot{\mathbf{r}}_{N}$ should be nonnegative as well, namely, $\dot{\mathbf{r}}_{N} \geq 0$. If $\dot{\mathbf{r}}_{N}>0$, it indicates that the impact process terminates and accordingly the contact force will vanish as well. And if $\dot{\mathbf{r}}_{N}=0$, the robot and the ground will keep contact, namely, $\boldsymbol{\Lambda}_{N} \geq 0$. Hence, there is a linear complementarity problem (LCP) existing in the conditions of impact process as follows:

$$
\dot{\mathbf{r}}_{N} \geq 0, \quad \boldsymbol{\Lambda}_{N} \geq 0, \quad \dot{\mathbf{r}}_{N} \cdot \boldsymbol{\Lambda}_{N}=0 .
$$

Therefore, if $\mathbf{J}_{\mathrm{TU}} \mathbf{M}^{-1} \mathbf{J}_{\mathrm{TU}}^{T}(\mathbf{n}-\mu \boldsymbol{\tau})$, namely, the coefficient matrix of $\Lambda_{N}$, is a positive definite matrix, the unique solution of $\dot{\mathbf{r}}$ and $\boldsymbol{\Lambda}_{N}$ can be obtained by uniting (21) and (23). Accordingly, the velocity of the robot as the impact completes can be solved by

$$
\dot{\mathbf{q}}_{E}=\mathbf{M}^{-1}\left(\mathbf{w}_{\tau} \cdot \Lambda_{\tau}-\mathbf{w}_{N} \cdot \Lambda_{N}\right)+\dot{\mathbf{q}}_{A} .
$$

4.3.1. USI Process. In USI process, the coefficient of friction $\mu$ is always $\mu_{d}$. By referring to (21) (24), as well as replacing the $t$ with intervals $\left[t_{A}, t_{C}\right]$ and $\left[t_{C}, t_{E}\right]$, the dynamics model of USI process can be expressed by the following.

(a) Compression phase:

$$
\begin{gathered}
\dot{\mathbf{r}}_{C}=\mathbf{J}_{\mathrm{TU}} \mathbf{M}^{-1}\left(\mathbf{w}_{N}-\mathbf{w}_{\tau} \cdot \mu_{d}\right) \boldsymbol{\Lambda}_{N, A C}+\dot{\mathbf{r}}_{A}, \\
\mathbf{n}^{T} \dot{\mathbf{r}}_{C} \geq 0, \quad \boldsymbol{\Lambda}_{N, A C} \geq 0, \quad \mathbf{n}^{T} \dot{\mathbf{r}}_{C} \cdot \boldsymbol{\Lambda}_{N, A C}=0 .
\end{gathered}
$$

(b) Expansion phase:

$$
\begin{gathered}
\dot{\mathbf{r}}_{E}=\mathbf{J}_{\mathrm{TU}} \mathbf{M}^{-1}\left(\mathbf{w}_{N}-\mathbf{w}_{\tau} \cdot \mu_{d}\right)\left(\boldsymbol{\Lambda}_{N, S}+\varepsilon_{P} \cdot \boldsymbol{\Lambda}_{N, A C}\right)+\dot{\mathbf{r}}_{C}, \\
\mathbf{n}^{T} \dot{\mathbf{r}}_{E} \geq 0, \boldsymbol{\Lambda}_{N, S} \geq 0, \quad \mathbf{n}^{T} \dot{\mathbf{r}}_{E} \cdot \boldsymbol{\Lambda}_{N, S}=0, \\
\boldsymbol{\Lambda}_{N, C E}=\boldsymbol{\Lambda}_{N, S}+\varepsilon_{P} \cdot \boldsymbol{\Lambda}_{N, A C},
\end{gathered}
$$


where $\dot{\mathbf{r}}_{A}, \dot{r}_{C}$, and $\dot{\mathbf{r}}_{E}$ are the values of $\dot{\mathbf{r}}$ at $t_{A}, t_{C}$, and $t_{E}$, respectively, $\boldsymbol{\Lambda}_{N, A C}$ and $\boldsymbol{\Lambda}_{N, C E}$ are the normal impulses of the compression phase and expansion phase, respectively, $\varepsilon_{P}$ is the coefficient of restitution which is the ratio of $\Lambda_{N, A C}$ to $\boldsymbol{\Lambda}_{N, C E}$, and $\boldsymbol{\Lambda}_{N, S}$ is the corrected impulse needed for preventing the robot and the ground from penetrating each other [25].

4.3.2. VI Process. If the status of impact is viscous, $\dot{\mathbf{r}}_{\tau}$ will remain unchanged once it decreases to 0 . Letting the impact process in the interval $\left[t_{1}, t_{2}\right]$ be viscous, (21) can be expressed by

$$
\mathbf{J}_{\mathrm{TU}} \mathbf{M}^{-1} \mathbf{J}_{\mathrm{TU}}^{T}\left(\mathbf{n}-\mu_{s} \boldsymbol{\tau}\right) \boldsymbol{\Lambda}_{N}=0 .
$$

By referring to (23), $\boldsymbol{\Lambda}_{N}$ must satisfy $\boldsymbol{\Lambda}_{N}>0$ in (27). As such, by solving (27), $\mu_{s}$ under the status of viscous impact $\left(\dot{\mathbf{r}}_{\tau}(t)=\right.$ $0)$ can be expressed by

$$
\mu_{s}=\frac{\boldsymbol{\tau}^{T} \mathbf{J}_{\mathrm{TU}} \mathbf{M}^{-1} \mathbf{J}_{\mathrm{TU}}^{T} \mathbf{n}}{\boldsymbol{\tau}^{T} \mathbf{J}_{\mathrm{TU}} \mathbf{M}^{-1} \mathbf{J}_{\mathrm{TU}}^{T} \boldsymbol{\tau}} .
$$

In VI process and RSI process, whether $t_{L}$, the time $\dot{r}_{\tau}(t)$ decreases to 0 , is within the compression phase $\left(t_{L} \in\left[t_{A}, t_{C}\right]\right)$ or the expansion phase $\left(t_{L} \in\left[t_{C}, t_{E}\right]\right)$, it will derive the different LCP of the compression and expansion phases.

(i) $t_{L}$ within Compression Phase $\left(t_{L} \in\left[t_{A}, t_{C}\right]\right)$. If $\Lambda_{N, A L} \leq$ $\Lambda_{N, A C}, t_{L} \in\left[t_{A}, t_{C}\right]$. By referring to (21) (24), the dynamics model of VI process can be expressed by the following.

(a) Compression phase:

$$
\begin{aligned}
\dot{\mathbf{r}}_{C}= & \mathbf{J}_{\mathrm{TU}} \mathbf{M}^{-1} \\
& \times\left[\mathbf{w}_{\tau}\left(\mu_{s}-\mu_{d}\right) \Lambda_{N, A L}+\left(\mathbf{w}_{N}-\mathbf{w}_{\tau} \cdot \mu_{s}\right) \boldsymbol{\Lambda}_{N, A C}\right]+\dot{\mathbf{r}}_{A}, \\
& \mathbf{n}^{T} \dot{\mathbf{r}}_{C} \geq 0, \quad \boldsymbol{\Lambda}_{N, A C} \geq 0, \quad \mathbf{n}^{T} \dot{\mathbf{r}}_{C} \cdot \boldsymbol{\Lambda}_{N, A C}=0 .
\end{aligned}
$$

(b) Expansion phase:

$$
\begin{gathered}
\dot{\mathbf{r}}_{E}=\mathbf{J}_{\mathrm{TU}} \mathbf{M}^{-1}\left(\mathbf{w}_{N}-\mathbf{w}_{\tau} \cdot \mu_{s}\right)\left(\boldsymbol{\Lambda}_{N, S}+\varepsilon_{P} \cdot \boldsymbol{\Lambda}_{N, A C}\right)+\dot{\mathbf{r}}_{C} \\
\mathbf{n}^{T} \dot{\mathbf{r}}_{E} \geq 0, \boldsymbol{\Lambda}_{N, S} \geq 0, \quad \mathbf{n}^{T} \dot{\mathbf{r}}_{E} \cdot \boldsymbol{\Lambda}_{N, S}=0 \\
\boldsymbol{\Lambda}_{N, C E}=\boldsymbol{\Lambda}_{N, S}+\varepsilon_{P} \cdot \boldsymbol{\Lambda}_{N, A C}
\end{gathered}
$$

(ii) $t_{L}$ within Expansion Phase $\left(t_{L} \in\left[t_{C}, t_{E}\right]\right)$. Likewise, if $\Lambda_{N, A L}>\Lambda_{N, A C}, t_{L} \in\left[t_{C}, t_{E}\right]$; namely, $t_{L}$ occurs within the expansion phase. Therefore, by referring to (21) (24), the dynamics model of the compression phase in VI process can be obtained as follows.

(a) Compression phase:

$$
\begin{gathered}
\dot{\mathbf{r}}_{C}=\mathbf{J}_{\mathrm{TU}} \mathbf{M}^{-1}\left(\mathbf{w}_{N}-\mathbf{w}_{\tau} \cdot \mu_{d}\right) \boldsymbol{\Lambda}_{N, A C}+\dot{\mathbf{r}}_{A}, \\
\mathbf{n}^{T} \dot{\mathbf{r}}_{C} \geq 0, \quad \boldsymbol{\Lambda}_{N, A C} \geq 0, \quad \mathbf{n}^{T} \dot{\mathbf{r}}_{C} \cdot \boldsymbol{\Lambda}_{N, A C}=0 .
\end{gathered}
$$

During the expansion phase, $\dot{r}_{\tau}(t)$ decreases from $\dot{r}_{\tau}\left(t_{C}\right)$ to 0 over the interval $\left[t_{C}, t_{L}\right]$. Based on the law of conservation of momentum, the normal impulse generated along the expansion phase can be obtained by $\Lambda_{N, C L}=-1 / E_{\tau} \dot{r}_{\tau}\left(t_{C}\right)$. Accordingly, the dynamics model of the expansion phase in VI process can be obtained as follows.

(b) Expansion phase:

$$
\begin{gathered}
\dot{\mathbf{r}}_{E}=\dot{\mathbf{r}}_{C}+\mathbf{J}_{\mathrm{TU}} \mathbf{M}^{-1} \mathbf{w}_{\tau} \cdot\left(\mu_{s}-\mu_{d}\right) \boldsymbol{\Lambda}_{N, C L} \\
+\mathbf{J}_{\mathrm{TU}} \mathbf{M}^{-1}\left(\mathbf{w}_{N}-\mathbf{w}_{\tau} \cdot \mu_{s}\right)\left(\boldsymbol{\Lambda}_{N, S}+\varepsilon_{P} \cdot \boldsymbol{\Lambda}_{N, A C}\right), \\
\mathbf{n}^{T} \dot{\mathbf{r}}_{E} \geq 0, \boldsymbol{\Lambda}_{N, S} \geq 0, \quad \mathbf{n}^{T} \dot{\mathbf{r}}_{E} \cdot \boldsymbol{\Lambda}_{N, S}=0, \\
\boldsymbol{\Lambda}_{N, C E}=\boldsymbol{\Lambda}_{N, S}+\varepsilon_{P} \cdot \boldsymbol{\Lambda}_{N, A C} .
\end{gathered}
$$

4.3.3. RSI Process. As discussed above, in RSI process, whether $t_{L} \in\left[t_{A}, t_{C}\right]$ or $t_{L} \in\left[t_{C}, t_{E}\right]$, it will derive the different LCP of the compression and expansion phases.

(iii) $t_{L}$ within Compression Phase $\left(t_{L} \in\left[t_{A}, t_{C}\right]\right)$. If $\Lambda_{N, A L}<$ $\Lambda_{N, A C}, t_{L} \in\left[t_{A}, t_{C}\right]$, whereupon the dynamics models of the compression and expansion phases can be expressed by the following.

(a) Compression phase:

$$
\begin{aligned}
\dot{\mathbf{r}}_{C}= & -2 \mathbf{J}_{\mathrm{TU}} \mathbf{M}^{-1} \mathbf{w}_{\tau} \mu_{d} \boldsymbol{\Lambda}_{N, A L}+\dot{\mathbf{r}}_{A} \\
& +\mathbf{J}_{\mathrm{TU}} \mathbf{M}^{-1}\left(\mathbf{w}_{N}+\mathbf{w}_{\tau} \cdot \mu_{d}\right) \boldsymbol{\Lambda}_{N, A C}, \\
\mathbf{n}^{T} \dot{\mathbf{r}}_{C} \geq & 0, \boldsymbol{\Lambda}_{N, A C} \geq 0, \quad \mathbf{n}^{T} \dot{\mathbf{r}}_{C} \cdot \boldsymbol{\Lambda}_{N, A C}=0 .
\end{aligned}
$$

(b) Expansion phase:

$$
\begin{aligned}
& \dot{\mathbf{r}}_{E}= \mathbf{J}_{\mathrm{TU}} \mathbf{M}^{-1}\left(\mathbf{w}_{N}+\mathbf{w}_{\tau} \cdot \mu_{d}\right) \\
& \times\left(\boldsymbol{\Lambda}_{N, S}+\varepsilon_{P} \cdot \boldsymbol{\Lambda}_{N, A C}\right)+\dot{\mathbf{r}}_{C}, \\
& \mathbf{n}^{T} \dot{\mathbf{r}}_{E} \geq 0, \boldsymbol{\Lambda}_{N, S} \geq 0, \quad \mathbf{n}^{T} \dot{\mathbf{r}}_{E} \cdot \boldsymbol{\Lambda}_{N, S}=0 .
\end{aligned}
$$

(iv) $t_{L}$ within Expansion Phase $\left(t_{L} \in\left[t_{C}, t_{E}\right]\right)$. Likewise, if $\Lambda_{N, A L}>\Lambda_{N, A C}, t_{L} \in\left[t_{C}, t_{E}\right]$, whereupon the dynamics model of the compression phase in RSI process can be obtained as follows.

(a) Compression phase:

$$
\begin{gathered}
\dot{\mathbf{r}}_{C}=\mathbf{J}_{\mathrm{TU}} \mathbf{M}^{-1}\left(\mathbf{w}_{N}-\mathbf{w}_{\tau} \cdot \mu_{d}\right) \boldsymbol{\Lambda}_{N, A C}+\dot{\mathbf{r}}_{A}, \\
\mathbf{n}^{T} \dot{\mathbf{r}}_{C} \geq 0, \quad \boldsymbol{\Lambda}_{N, A C} \geq 0, \quad \mathbf{n}^{T} \dot{\mathbf{r}}_{C} \cdot \boldsymbol{\Lambda}_{N, A C}=0 .
\end{gathered}
$$

During the expansion phase in RSI process, $\dot{r}_{\tau}(t)$ decreases from $\dot{r}_{\tau}\left(t_{C}\right)$ to 0 over the interval $\left[t_{C}, t_{L}\right]$. Likewise, the normal impulse generated along the expansion phase can be expressed by $\Lambda_{N, C L}=-1 / E_{\tau} \dot{r}_{\tau}\left(t_{C}\right)$. Correspondingly, the dynamics model of the expansion phase in RSI process can be obtained as follows. 
(b) Expansion phase:

$$
\begin{aligned}
\dot{\mathbf{r}}_{E}= & \dot{\mathbf{r}}_{C}-2 \mathbf{J}_{\mathrm{TU}} \mathbf{M}^{-1} \mathbf{w}_{\tau} \cdot \mu_{d} \boldsymbol{\Lambda}_{N, C L} \\
& +\mathbf{J}_{\mathrm{TU}} \mathbf{M}^{-1}\left(\mathbf{w}_{N}+\mathbf{w}_{\tau} \cdot \mu_{d}\right) \\
& \times\left(\boldsymbol{\Lambda}_{N, S}+\varepsilon_{P} \cdot \boldsymbol{\Lambda}_{N, A C}\right), \\
\mathbf{n}^{T} \dot{\mathbf{r}}_{E} \geq 0, & \boldsymbol{\Lambda}_{N, S} \geq 0, \quad \mathbf{n}^{T} \dot{\mathbf{r}}_{E} \cdot \boldsymbol{\Lambda}_{N, S}=0 .
\end{aligned}
$$

\section{Computational Results and Their Analysis}

5.1. Computational Method for the Dynamics Models. As the dynamics models of the flying and landing process derived above, the dynamics model of flying process is relatively uncomplicated to be solved, which is determined by the dynamics of take-off process. Hence, this paper mainly presents the computational method for the dynamics of landing impact process, which is summarized as follows.

(1) Define $\mu_{d}, \varepsilon_{p}$, and the structural parameters of the robot; obtain $\mathbf{J}_{\mathrm{TU}}$ and $\dot{\mathbf{r}}\left(t_{A}\right)$ by solving the dynamics of flying process.

(2) Calculate the values of $\Lambda_{N, A L}, \Lambda_{N, A C}, E_{\tau}, E_{n}, E_{\tau}^{\prime}$, and $E_{n}^{\prime}$, and then accordingly classify the type of impact process.

(3) Solve $\dot{\mathbf{r}}\left(t_{E}\right)$, the relative velocity between the robot and the ground after landing impact process, by using applicable $\mu_{s}$ and dynamics models.

(4) Based on $\dot{\mathbf{r}}\left(t_{E}\right)$, calculate $\dot{\mathbf{q}}\left(t_{E}\right)$, the generalized velocity after impact process, and then accordingly obtain the motion characteristics of the robot after landing impact process.

(5) If $\dot{\mathbf{q}}\left(t_{E}\right)$ is not small enough, it means that the robot will bounce off. In this case, the steps presented above will repeat, by considering the motion characteristics after the previous landing impact process as the initial motion characteristics of the next flying process.

5.2. Computational and Corresponding Experimental Results. To compare with prototype experiments, in accordance with the experimental conditions of prototype, the initial motion characteristics of landing impact of the proposed robot are given as follows:

(1) attitude angles of the parts of the robot (foot, shank, thigh, trunk, and 2 connecting links) are $3.3^{\circ}, 30.9^{\circ}$, $81.6^{\circ}, 9.8^{\circ}, 38.5^{\circ}$, and $87.4^{\circ}$, respectively;

(2) $\dot{\mathbf{r}}_{O S}=[1.4873,2.4378]($ Unit: $\mathrm{m} / \mathrm{s}), \omega=1.29 \mathrm{rad} / \mathrm{s}$.

Let $\varepsilon_{p}=0.5$ and $\mu_{d}=0.8$, and let the inertia tensor of the robot $\mathbf{I}_{S}[24]$ be

$$
\mathbf{I}_{S}=\left[\begin{array}{ccc}
0.0052 & 0 & 0 \\
0 & 0.0050 & 0 \\
0 & 0 & 0.0011
\end{array}\right]
$$

The computation is implemented by plugging the structural parameters presented in [24] and the initial motion
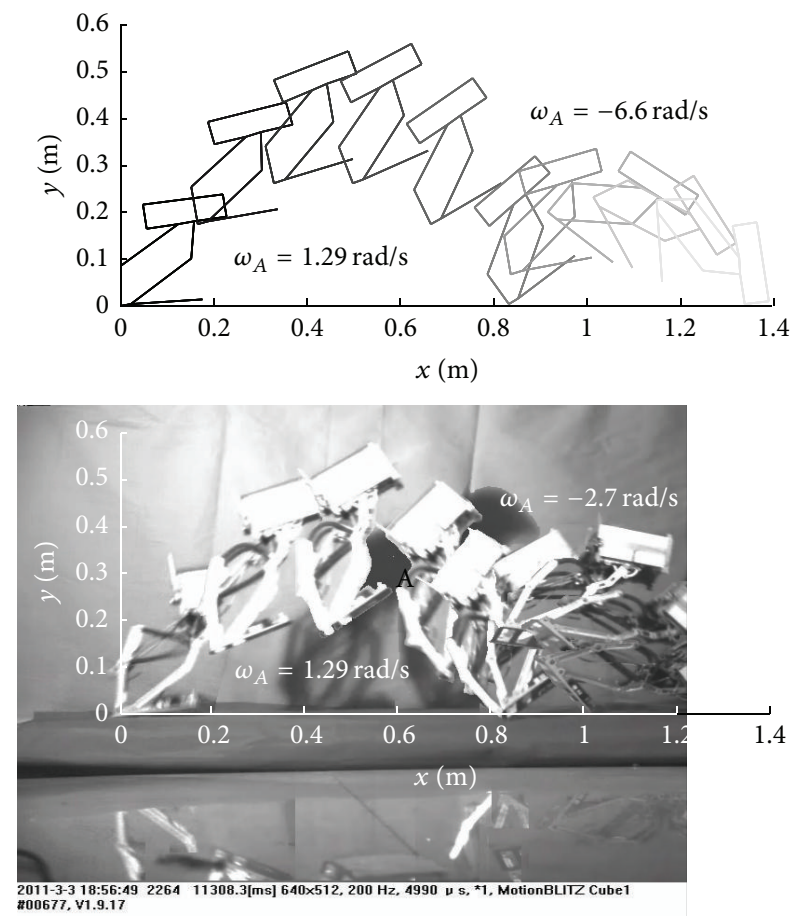

FIGURE 4: Flying and landing stance of the robot based on both of computational and experimental results, where timing starts as soon as the robot is about to leave the ground.

parameters above into the flying and landing dynamics equations and their computational methods. And the experiments of the prototype are carried out in an indoor environment and filmed by the high speed camera system MotionBLITZ Cube, at 120 frames per second (the video file titled "hopping_video.wmv," which can be found in the Supplementary Materials available online at http://dx.doi.org/10.1155/2014/374290). Figure 4 shows the stance of the robot in flying and landing process by comparing the computational and experimental results.

As shown in Figure 4, it is obvious that the flying and landing stance of the computational results are in accordance with the experimental results of the prototype. After take-off, the robot flies steadily, with the COM moving in a parabolic trajectory. As the robot is landing, the foot is the first in touchdown; then, the landing impact commences. After the impact process, the robot bounces off, tumbles forward, and then slightly alternates impact and bounce until it stops on the ground. By using a high speed video motion analysis system, the rotation angle of the prototype can be measured. Figure 4 presents the contrast of the computational data and experimental data on the rotational velocity of $S$ after first landing impact, where the computational result is $6.6 \mathrm{rad} / \mathrm{s}$ and the experimental result is $2.7 \mathrm{rad} / \mathrm{s}$. It is obvious that the computational values are larger than the experimental values, which is mainly caused by the difference between the given computation conditions and practical experiment conditions as follows.

(1) As shown in Figure 1, each component part of the proposed robot is of irregular shape. Although, without 
loss of generality, the dynamics model (as shown in Figure 2) assumes the COM of parts of the robot can be located at an arbitrary position, the practical mass distribution of the prototype is difficult to be measured.

(2) The ground in the prototype experiments is mixed by three layers of materials: the surface is woollen cloth, the middle is sponge, and the bottom is the wooden board. Therefore, the practical contact parameters are difficult to be obtained.

(3) Since the relative position and attitude between the high speed camera system and the robot may be improper, it will also cause errors by measuring the video snapshots to obtain the experiment data.

As discussed above, in summary, the coincidence of the computational and experimental results on flying and landing stance positively verifies the availability of the dynamics modelling above and its computation that has general applicability to the motion prediction, performance analysis, and landing stability study for intermittent hopping robots. Nevertheless, aimed at obtaining computational results more consistent with practical situation, the structural parameters, initial motion parameters, and contact condition parameters should be in accordance with practical conditions.

\section{Analysis of Influence Factors for Landing Impact}

Impact will inevitably occur when hopping robot is landing. Hence, to solve the landing stability problems and especially prevent the internal instruments equipped in trunk from being destroyed by impact, not only how to damp the impact but also how to avoid impact between the trunk of the robot and the ground should be considered. As shown in Figure 4, the robot flies stably and the rear end of its sole is the first to drop to ground when landing, which is in accordance with the moving stance of the kangaroos [27], that is, stretching its leg forward when landing. It is helpful for landing stability, because the foot first dropping to ground can practicably prevent the trunk, equipped with solar cells, controller, wireless vision system, and so forth, from sharply impacting the ground. However, when the robot bounces off the ground after landing, it tumbles forward. In the next landing impact process, the trunk of the robot is the first to impact the ground. Although, in the following process, alternating landing impact and bounce, the velocity of the robot and the height of bounces gradually reduce until the robot stops on the ground, the internal instruments still may be destroyed by impact happening in bounce processes.

As the dynamics model of landing impact given above, when the robot impacts the ground, the robot is subjected to gravity $\mathbf{G}$ on $S$ as well as impact force $\mathbf{F}_{C}$ on $U_{1}$. Since $\mathbf{F}_{C}$ does not act on $S$, it will generate a torque $L_{F C}$, which changes the angular momentum of the robot. According to the assumptions of the rigid impact model $[25,26]$, the inertia tensor of the robot $\mathbf{I}_{S}$ keeps unchanged during landing impact, so that the rotational velocity of $S$ is changed. Hence,
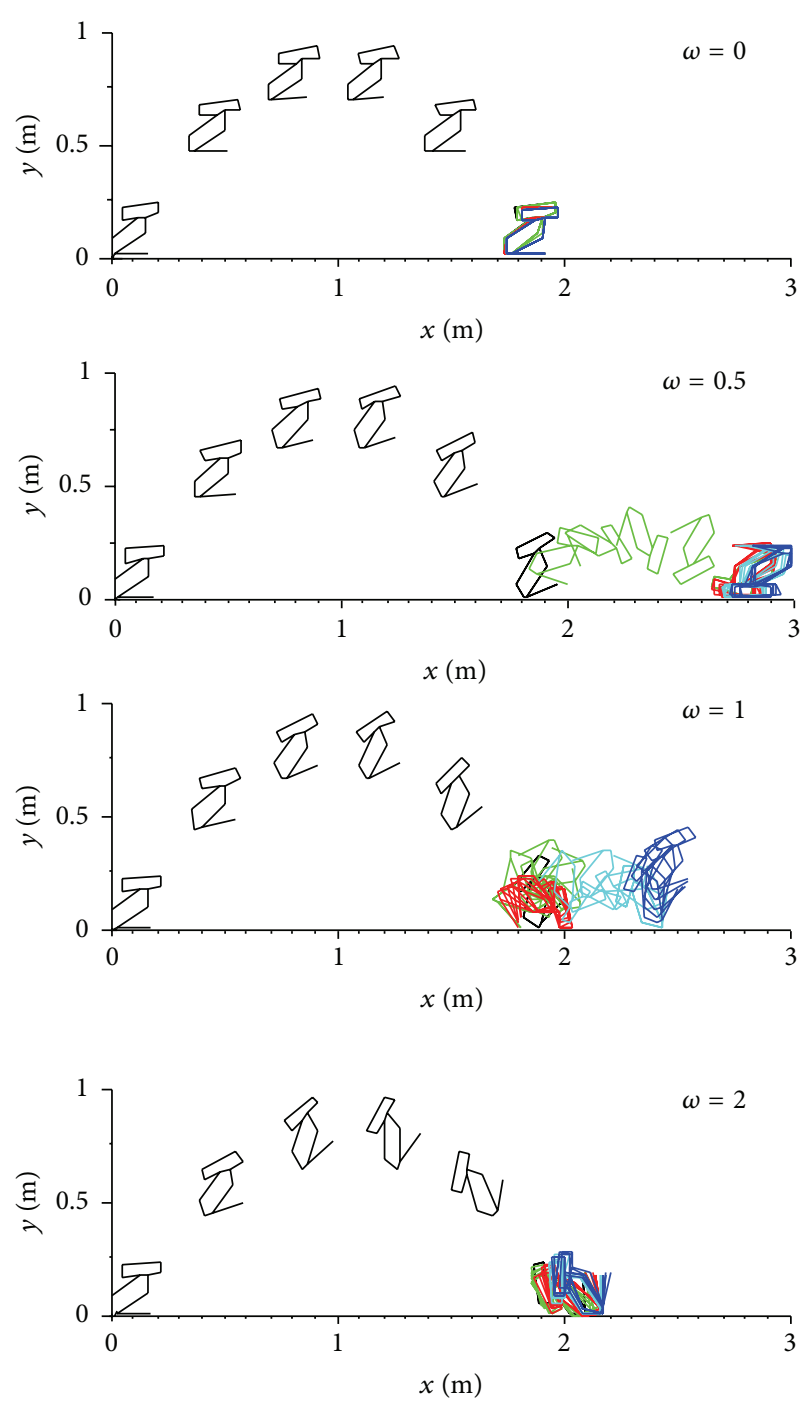

FIGURE 5: Effect of the kinematical parameter $\omega_{A}$ on landing impact.

as shown in Figure 4, the robot tumbles forward after landing impact.

Likewise, as the dynamics model given above, the motion stance of the robot after landing impact is mainly influenced by the kinematical parameters before landing impact (mainly the rotational velocity of $S$ when landing impact occurs, $\omega_{A}$ ), the coefficient of sliding friction $\mu_{d}$ and the coefficient of restitution $\varepsilon_{p}$. Therefore, the landing stances under different initial moving status can be obtained as shown in Figure 5 by specifying $\omega_{A}$ as a set of given values $[0,0.5,1,2]$ (unit: $\mathrm{rad} / \mathrm{s}$ ) and keeping other parameters unchanged. It should be noted that the construcal parameters and initial motion conditions in this section (analysis of influence factors) are in accordance with the computational results in [24].

As shown in Figure 5, if $\omega_{A}=0, L_{F C}$ should be zero and $\mathbf{I}_{S}$ should be zero during take-off process. That is, the motion stance of the robot in flying process keeps unchanged, so that the contacting points between the robot and the ground in landing process and in take-off process should lie in the same position on the robot. Consequently, $L_{F C}$ should be zero 


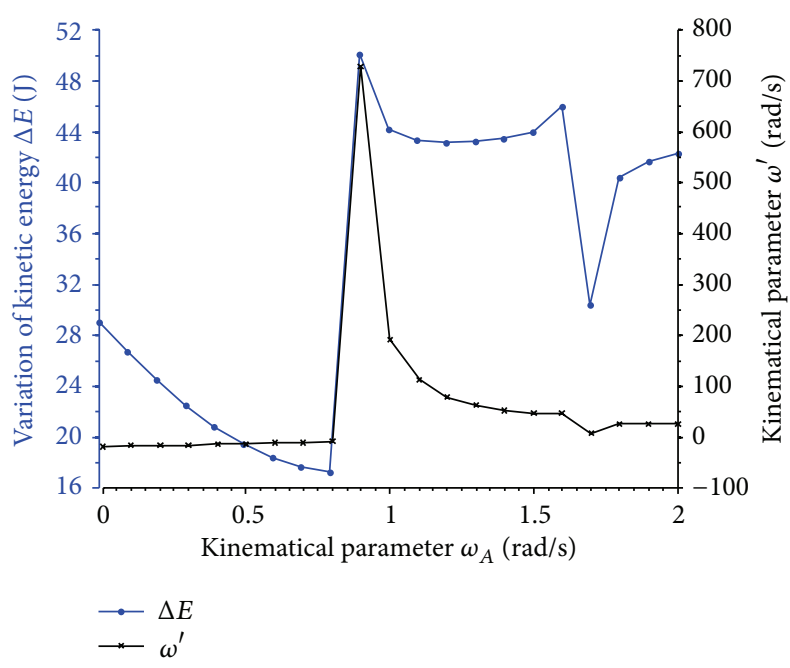

FIGURE 6: Effect of $\omega_{A}$ on the kinetic energy loss $\Delta E$ and the rotation velocity $\omega^{\prime}$.

during landing impact; that is, $\mathbf{I}_{S}$ keeps unchanged $\left(\mathbf{I}_{S}=0\right)$, so that the robot lands stably without tumbling. However, as presented in [24], the configuration of the robot and the contacting point is ever-changing during take-off, so that $\omega_{A}=0$ is an extremely ideal case and difficult to achieve. Therefore, if $\omega_{A} \neq 0$, the bigger $\omega_{A}$, the larger the rotational angle of the robot during flying process. Since $S$ lies in the trunk of the robot [24], the more the robot tumbles when landing, the more the $S$ is close to the ground. In the case that $S$ is close to the ground and rotational velocities of parts of the robot relative to $S$ are large, the parts of the robot may sharply impact the ground again in a very short period of time during the robot landing impacts and bounces. That is, after a landing impact process, continuous and sharp impact near the contacting point will happen to the robot, as in the case $\omega_{A}=1$ shown in Figure 5.

Shown in Figure 5, the effect of $\omega_{A}$ on the motion stance is only analysed intuitively. As discussed above, the landing stability of the robot is intuitively performed by the height of bounces and the rotation velocity after landing impact. It is known that the height of bounces depend on how much the kinetic energy loss is in landing impact process. The kinetic energy of the robot can be expressed by

$$
E=\frac{1}{2}\left(\mathbf{M} \dot{q}_{1}^{2}+\mathbf{M} \dot{q}_{2}^{2}+\mathbf{I}_{S} \dot{q}_{3}^{2}\right)
$$

where $q=\left[q_{1}, q_{2}, q_{3}\right]^{T}=[x, y, \alpha]^{T}$ is the generalized coordinates.

By taking complete differential of (38), the kinetic energy loss of the robot due to sudden change of velocities in landing impact can be obtained by

$$
\Delta E=\frac{1}{2}\left(\mathbf{M} \dot{q}_{1} \Delta \dot{q}_{1}+\mathbf{M} \dot{q}_{2} \Delta \dot{q}_{2}+\mathbf{I}_{S} \dot{q}_{3} \Delta \dot{q}_{3}\right)
$$

where $q_{1}, q_{2}$, and $q_{3}$ satisfy $\Delta \dot{\mathbf{q}}=\dot{\mathbf{q}}_{E}-\dot{\mathbf{q}}_{A}$.

By specifying $\omega_{A}$ as a set of given values and keeping other parameters unchanged, the kinetic energy loss $\Delta E$ and

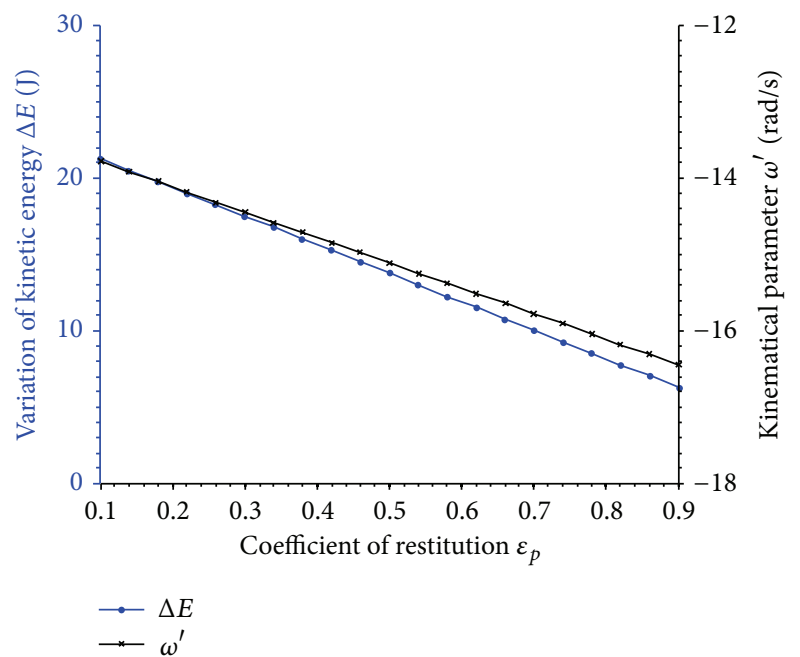

FIGURE 7: Effect of $\varepsilon_{p}$ on the kinetic energy loss $\Delta E$ and the rotation velocity $\omega^{\prime}$.

rotation velocity $\omega^{\prime}$ after the first landing impact process under different initial moving status can be obtained as shown in Figure 6. When $\omega_{A}<0.8$, as $\omega_{A}$ increases, $\omega^{\prime}$ increases gradually, while $\Delta E$ decreases. It indicates that the height of bounces and rotation velocities increase as $\omega_{A}$ increases. When $\omega_{A}>1, \Delta E$ is large, and $\omega^{\prime}$ decreases from large values. The height of bounce is small; however the rotation velocity is large, which indicates that continuous and sharp impact near the contacting point will happen to the robot after a landing impact process. As $\omega_{A}$ is close to 1.7, both $\Delta E$ and $\omega^{\prime}$ have a sudden change. It is because the trunk of the robot is the first in touchdown by replacing the foot as shown in Figure 5. As discussed above, in summary, the smaller the $\omega_{A}$ is, the more the robot flies stably as well as landing stably after impact.

Likewise, the kinetic energy loss $\Delta E$ and rotation velocity $\omega^{\prime}$ after the first landing impact process under the conditions of different $\varepsilon_{p}$ can be obtained as shown in Figure 7. It is obvious that the larger $\varepsilon_{p}$, the larger the scalar values of $\omega^{\prime}$ and the smaller the $\Delta E$. Namely, the height of bounces and the times of bounces will increase as $\varepsilon_{p}$ increases. It indicates that the larger $\varepsilon_{p}$ is, the less beneficial to landing stability and damping impact it is. Since $\varepsilon_{p}$ is the ratio of the normal velocity of impact point before landing impact to the normal velocity of impact point after landing impact, the smaller it is, the larger the loss of the kinetic energy of the robot is during landing impact, such that the times of bounces are less. Hence, after the flying process, if the robot lands on the terrain whose $\varepsilon_{p}$ is smaller, the risk of landing impact destroying the robot reduces.

Figure 8 shows the kinetic energy loss $\Delta E$ and rotation velocity $\omega^{\prime}$ after the first landing impact process under the conditions of different $\mu_{d}$. As shown in Figure 8, along with $\mu_{d}$ increasing, both $\Delta E$ and the scalar values of $\omega^{\prime}$ decrease. When $\mu_{d}$ is smaller, the height of bounces becomes smaller, but the rotational velocities of the robot during bounces are larger. As the initial rotation velocities are large, referring to Figure 6, continuous and sharp impact near 


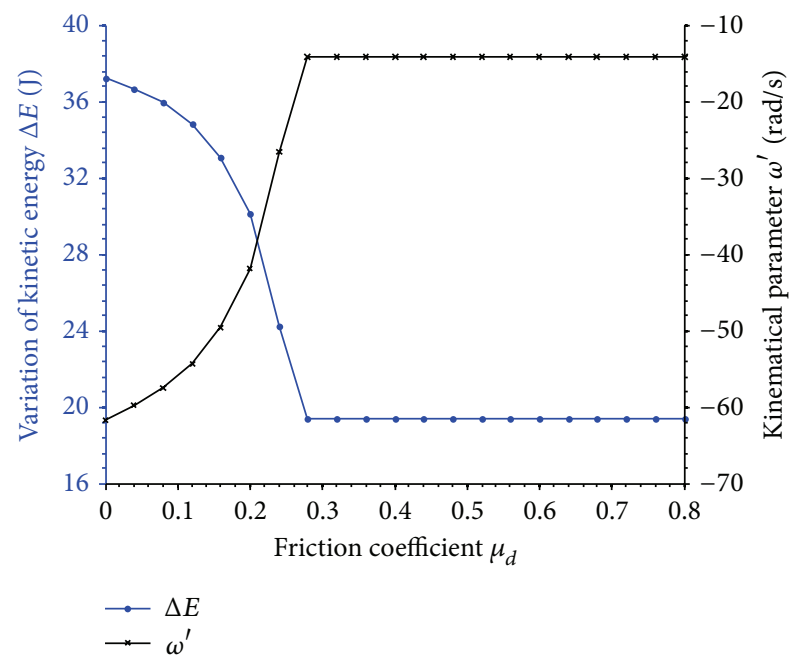

FIGURE 8: Effect of $\mu_{d}$ on the kinetic energy loss $\Delta E$ and the rotation velocity $\omega^{\prime}$.

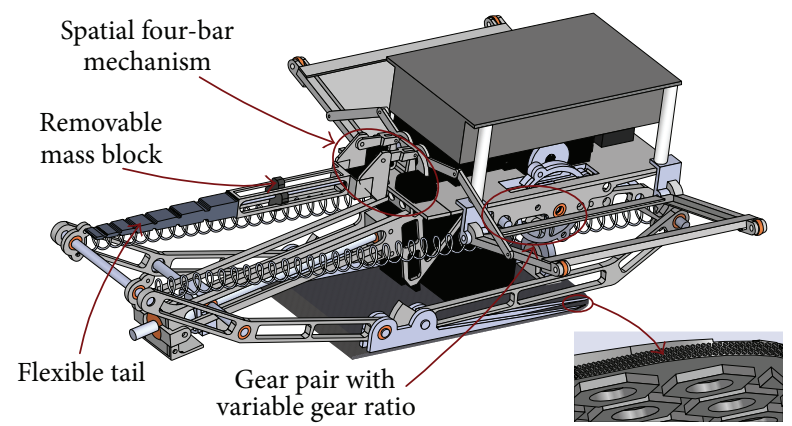

FIGURE 9: Three-dimensional model for the improved design of the proposed robot.

the contacting point will happen to the robot after a landing impact process. Therefore, as $\mu_{d}$ is larger, it is more beneficial to landing stability and damping impact. However, as shown in Figure 8, when $\mu_{d}$ is larger than $0.3, \Delta E$ and $\omega^{\prime}$ show little change. That is, $\mu_{d}$ has great influence on the robot if $\mu_{d}$ is small and, on the contrary, has little influence if $\mu_{d} \geq 0.3$.

To sum up, the fact that the rotational velocity of $S$ before landing impact occurs is smaller, the coefficient of restitution $\varepsilon_{p}$ is smaller, and the coefficient of sliding friction $\mu_{d}$ is larger is more conducive to improve landing stability of the robot and reduce the risk of the internal instruments of the robot destroyed by violent impact.

\section{An Improved Design for the Proposed Robot}

According to the results discussed above, aimed at improving the conditions of landing impact process, a technical design for the robot is proposed as shown in Figure 9.

The improved design can be summarized as follows.

(1) To Reduce $\omega_{A}$. Usually, in running and jumping process, people or animals can achieve a steady jumping and flying stance by adjusting their pose of jumping and rotation velocity of flying. The difference is that people keep flying steadily by swing of their arms and legs, and animals usually use their tails such as the kangaroos [27]. Therefore, bioinspired by the jumping characteristics of kangaroos, a steerable tail is designed as shown in Figure 9. The tail is driven by a spatial four-bar mechanism and two robotic servomotors. It can achieve arbitrarily spatial attitudes, which are able to satisfy the need for adjusting flying stance. In order to adjust the attitude of jumping, the gear pair at hip joint of the robot is improved by replacing with a gear pair with variable gear ratios. The improved robot can adjust its attitudes of jumping by steering the ratio of the gear pair.

Additionally, to enhance the adaptation of the tail, the rear tail is designed by a flexible mechanism. And a removable mass block is designed to adjust the rotational inertia of the tail as well.

(2) To Reduce $\varepsilon_{p}$. As shown in Figure 6, to reduce $\varepsilon_{p}$, it mainly depends on how to increase the kinetic energy loss $\Delta E$. Therefore, to reduce $\varepsilon_{p}$, it needs to reduce the stiffness and increase the damping of contact between the robot and the ground. Accordingly, the foot is designed by using porous and lightweight materials as shown in Figure 9.

(3) To Increase $\mu_{d}$. As shown in Figure 8 , when $\mu_{d}$ is larger, it is more beneficial to landing stability and damping impact. However, $\mu_{d}$ also does not necessarily have to be too large, because it has little influence on landing impact if $\mu_{d} \geq$ 0.3 . Therefore, the porous and lightweight materials of the foot presented above are designed as two layers as shown in Figure 9. The two layers of porous materials have horizontal and vertical cell structure, respectively. The internal layer is horizontal cell structure which is beneficial to absorb the landing impact energy. And the external layer is vertical cell structure which is beneficial to increase $\mu_{d}$.

The improved design presented above is beneficial to the mobility of intermittent hopping robots or other legged robots. However, in consideration of practicality, there are still some challenges as follows.

(1) Dynamics modelling of adjusting the jumping stance of the robot through real-time adjusting the attitude of the tail is difficult. Since the robot is a moving system in jumping process and the tail is with flexible structure, the dynamic model is a rigid flexible coupling system with floating reference.

(2) Since the period of single jumping process is very short, real-time adjusting of the tail in jumping process needs high speed and high torque actuators. This is difficult to achieve by micropower servomotors.

\section{Conclusions and Summary}

Based on the prior work presenting the mechanical design and take-off dynamics analysis of the proposed robot in [24], this paper concentrates on further study of the landing impact dynamics and its influence factors of the proposed robot, which is summarized as follows. 
(1) Multibody dynamics for the flying and landing oblique impact processes of the proposed robot is derived by using the impulse-momentum principle. The dynamics modelling involves the multibody configuration, mass distribution of the robot, and friction. The computational and experimental results show that the proposed dynamics models and their solution methods are available to reveal the practical flying and landing stances of the robot and have general applicability to the motion prediction and performance analysis of intermittent hopping robots as well.

(2) The effects of the initial status of landing, the coefficient of sliding friction, and the coefficient of restitution on landing impact are analysed. The results show that the smaller the rotational velocity of the robot before landing and the coefficient of restitution are and the larger the coefficient of sliding friction is, the more the robot is benefited to improve landing stability and reduce the damaged risk of the internal instruments.

(3) Based on the analysis of influence factors for landing impact, an improved design for the proposed robot is proposed. By adjusting the robot's attitude during the jumping and absorbing the impact energy during the landing, the technical design provides a possible solution for the landing stability of intermittent hopping robots or other legged robots.

\section{Conflict of Interests}

The authors declare that there is no conflict of interests regarding the publication of this paper.

\section{Acknowledgments}

This research is supported by National Natural Science Foundation of China (Grant no. 51405046), the Fundamental Research Funds for the Central Universities (Grant no. CDJZR13110004), and the Foundation for Sci \& Tech Research Project of Chongqing Science \& Technology Commission (Grant no. cstc2013jcsf70004).

\section{References}

[1] G. Carbone and M. Ceccarelli, "Legged robotic systems," in Cutting Edge Robotics, pp. 553-576, Pro Literatur, 2005.

[2] R. Armour, K. Paskins, A. Bowyer, J. Vincent, W. Megill, and R. Bomphrey, "Jumping robots: a biomimetic solution to locomotion across rough terrain," Bioinspiration and Biomimetics, vol. 2, no. 3, pp. S65-S82, 2007.

[3] M. H. Kaplan and H. Seifert, "Hopping transporters for lunar exploration," Journal of Spacecraft and Rockets, vol. 6, no. 3, pp. 917-922, 1969.

[4] K. Schilling and C. Jungius, "Mobile robots for planetary exploration," Control Engineering Practice, vol. 4, no. 4, pp. 513$524,1996$.
[5] S. Dubowsky, S. Kesner, J. S. Plante, and P. Boston, "Hopping mobility concept for search and rescue robots," Industrial Robot, vol. 35, no. 3, pp. 238-245, 2008.

[6] A. Sayyad, B. Seth, and P. Seshu, "Single-legged hopping robotics research-a review," Robotica, vol. 25, no. 5, pp. 587613, 2007.

[7] L. Bai, W.-J. Ge, X.-H. Chen, and M. Zhang, "Research on hopping robot for planetary exploration," Robot, vol. 31, no. 4, pp. 311-319, 2009.

[8] M. Kovač, M. Fuchs, A. Guignard, J.-C. Zufferey, and D. Floreano, "A miniature $7 \mathrm{~g}$ jumping robot," in Proceedings of the IEEE International Conference on Robotics and Automation (ICRA '08), pp. 373-378, IEEE, Pasadena, Calif, USA, May 2008.

[9] J. Zhao, G. Liu, J. Yan, and X. Zang, "Scout robot with wheelinghopping combination locomotion," Industrial Robot, vol. 36, no. 3, pp. 244-248, 2009.

[10] M. Wang, X. Z. Zang, J. Z. Fan, and J. Zhao, "Biological jumping mechanism analysis and modeling for frog robot," Journal of Bionic Engineering, vol. 5, no. 3, pp. 181-188, 2008.

[11] J. Burdick and P. Fiorini, "Minimalist jumping robots for celestial exploration," International Journal of Robotics Research, vol. 22, no. 7-8, pp. 653-674, 2003.

[12] P. Fiorini and J. Burdick, "The development of hopping capabilities for small robots," Autonomous Robots, vol. 14, no. 2-3, pp. 239-254, 2003.

[13] S. Shimoda, A. Wingart, K. Takahashi, T. Kubota, and I. Nakatani, "Microgravity hopping robot with controlled hopping and landing capability," in Proceedings of the IEEE/RSJ International Conference on Intelligent Robots and Systems, pp. 2571-2576, Piscataway, NJ, USA, October 2003.

[14] T. Yoshimitsu, "Development of autonomous rover for asteroid surface exploration," in Proceedings of the IEEE International Conference on Robotics and Automation, pp. 2529-2534, Piscataway, NJ, USA, May 2004.

[15] S. Dubowsky, S. Kesner, J.-S. Plante, and P. Boston, "Hopping mobility concept for search and rescue robots," Industrial Robot, vol. 35, no. 3, pp. 238-245, 2008.

[16] K. S. Kim, B. S. Kim, J. B. Song, and E. S. Kim, "Adjustable jumping angle-height mechanism for a jumping robot," in Proceedings of the Korean Society of Mechanical Engineering Annuals Spring \& Fall Conference, pp. 145-147, 2008.

[17] K.-S. Kim, B.-S. Kim, J.-B. Song, and C. H. Yim, "Mobility improvement of a jumping robot using conical spring with variable length endtip," Journal of Institute of Control, Robotics and Systems, vol. 15, no. 11, pp. 1108-1114, 2009.

[18] H. Tsukagoshi, M. Sasaki, A. Kitagawa, and T. Tanaka, "Jumping robot for rescue operation with excellent traverse ability," in Proceedings of the IEEE 12th International Conference on Advanced Robotics (ICAR '05), pp. 841-848, Piscataway, NJ, USA, July 2005.

[19] E. Watari, H. Tsukagoshi, A. Kitagawa, and T. Tanaka, "A higher casting and jump motions realized by robots using magnetic brake cylinder," Journal of Mechanisms and Robotics, vol. 3, no. 4, Article ID 041002, 2011.

[20] M. Kovač, M. Schlegel, J.-C. Zufferey, and D. Floreano, "Steerable miniature jumping robot," Autonomous Robots, vol. 28, no. 3, pp. 295-306, 2010.

[21] A. L. Desbiens, M. Pope, F. Berg, Z. E. Teoh, J. Lee, and M. Cutkosky, "Efficient jumpgliding: theory and design considerations," in Proceedings of the IEEE International Conference on Robotics and Automation (ICRA '13), pp. 4451-4458, Karlsruhe, Germany, May 2013. 
[22] Z. Z. Liu, J. Y. Zhu, H. T. Wu, and W. M. Xi, "Analysis of the landing impact and stability of the intermittent one legged hopping robot," Mechanical Science and Technology, vol. 23, no. 9, pp. 1068-1071, 2004.

[23] Y. Sato, E. Ohashi, and K. Ohnishi, "Impact force reduction for hopping robot," in Proceedings of the 31st Annual Conference of IEEE Industrial Electronics Society (IECON '05), pp. 1821-1826, November 2005.

[24] L. Bai, W. Ge, X. Chen, and R. Chen, "Design and dynamics analysis of a bio-inspired intermittent hopping robot for planetary surface exploration," International Journal of Advanced Robotic Systems, vol. 9, article 108, 2012.

[25] A. Peter and B. Hu, Advanced Contact Dynamics, Dongnan University Press, Nanjing, China, 2003.

[26] D. E. Stewart, "Rigid-body dynamics with friction and impact," SIAM Review, vol. 42, no. 1, pp. 3-39, 2000.

[27] R. M. Alexander and A. Vernon, "The mechanics of hopping by kangaroos (Macropodidae)," Journal of Zoology in London, vol. 177, no. 2, pp. 265-303, 1975. 


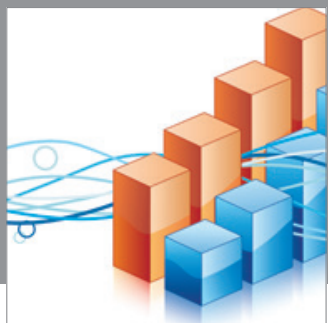

Advances in

Operations Research

mansans

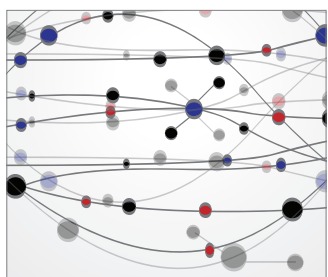

The Scientific World Journal
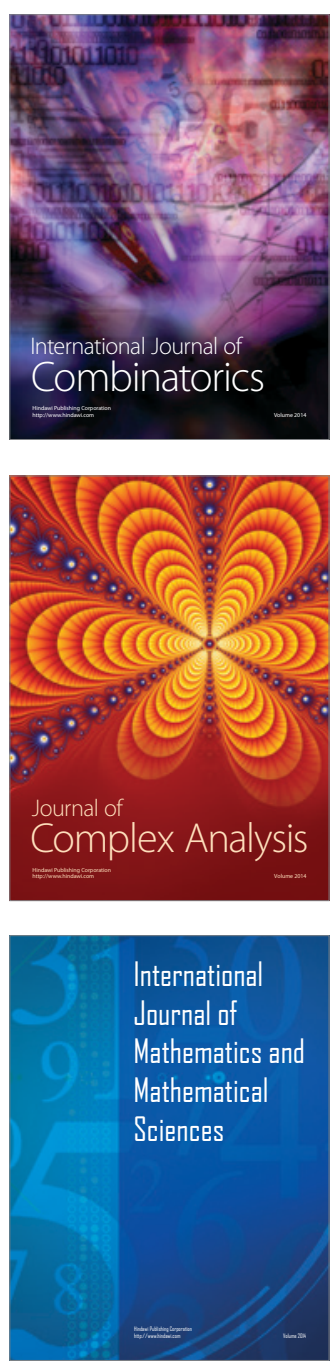
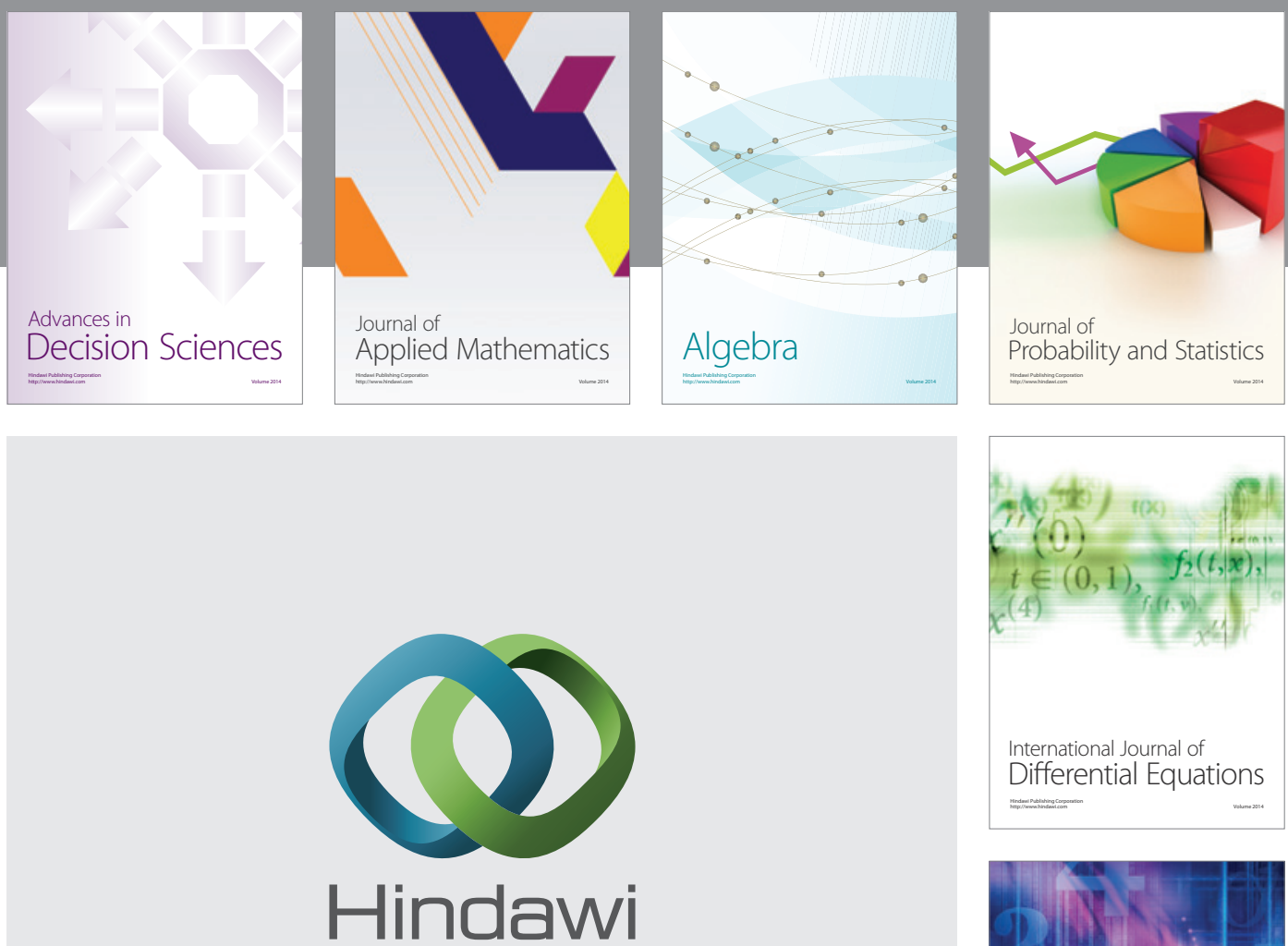

Submit your manuscripts at http://www.hindawi.com
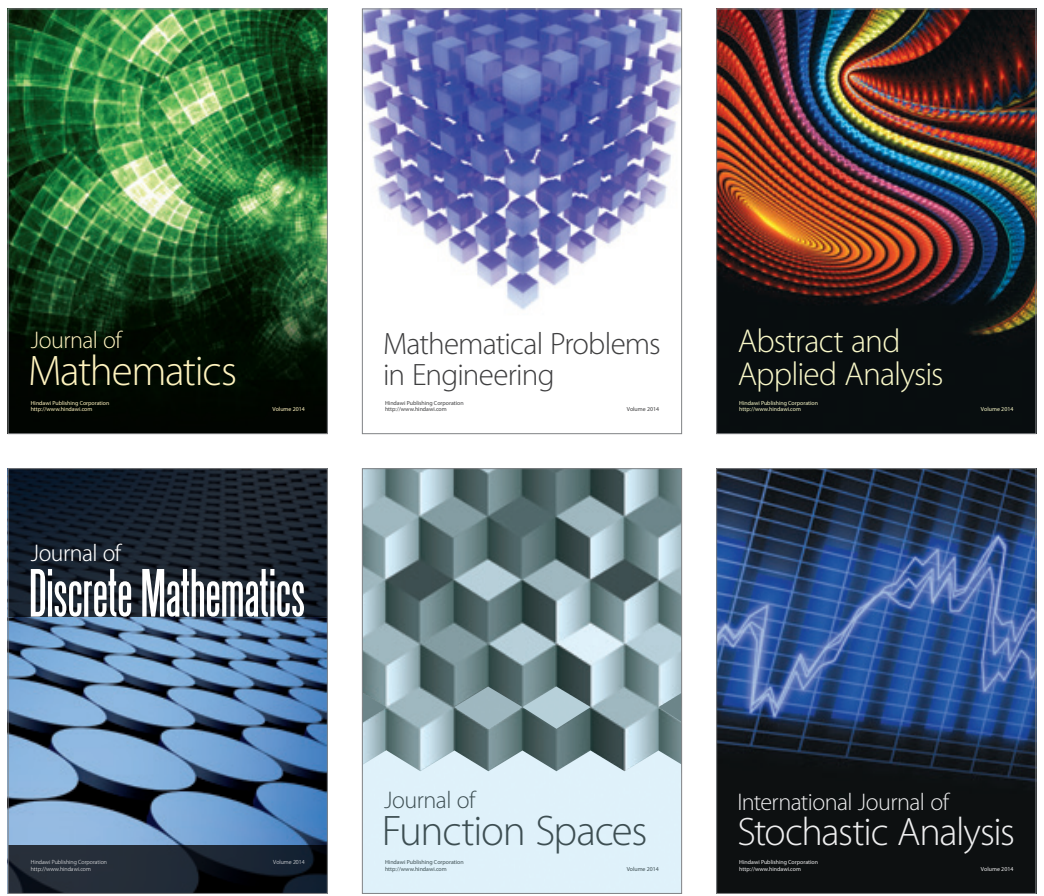

Journal of

Function Spaces

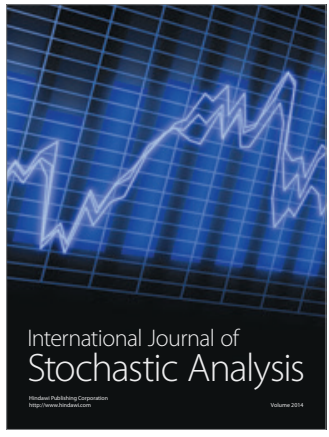

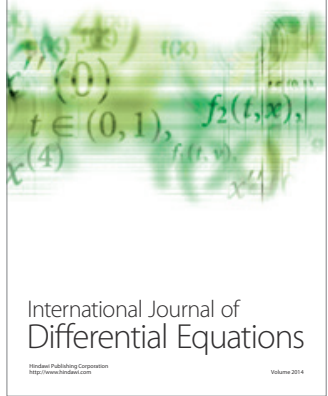
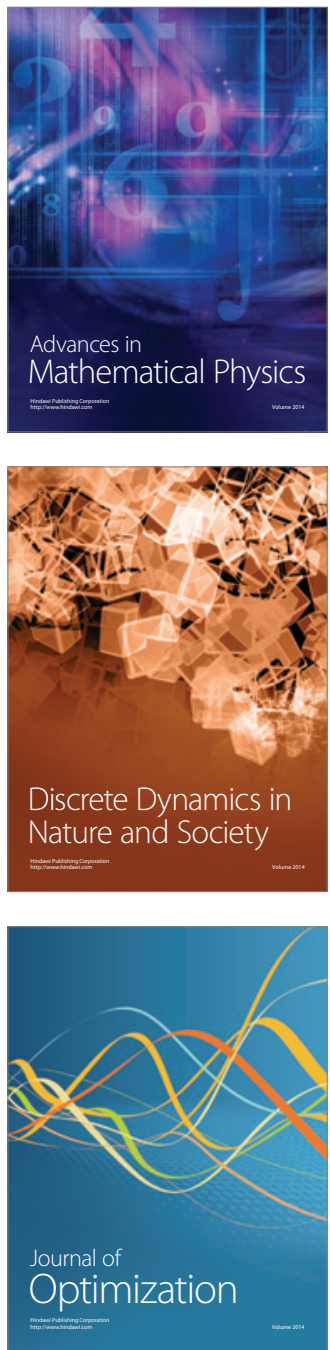